\title{
MODELAMIENTO MATEMÁTICO APLICADO A CONMINUCIÓN
}

\section{MATHEMATICAL MODELING APPLIED TO COMMINUTION}

\author{
Mercedes Aida Osorio Maza ${ }^{1}$, Zoila Emperatriz Ruiz Mostacero ${ }^{2}$, Edgar Ademar Pérez \\ Matos $^{3}$, Luis Alvarado Jaramillo ${ }^{4}$,
}

\begin{abstract}
RESUMEN
El modelamiento matemático aplicado a equipos destinados en el proceso de conminución de minerales (chancado, molienda), suelen ser herramientas tan importantes para llegar a simular y luego optimizar procesos metalúrgicos continuos (a nivel industrial); vale decir llegar a predecir el producto granulométrico de un cierto mineral tratado, conociendo tan solo lo del alimento (simulación). Obviamente el modelo almacenará intrínsecamente, inherentemente las características físicas del mineral (dureza, fractura) y operativas del equipo (aberturaCSS).Consiguiendo de esta manera mejorar el rendimiento del equipo en cuestión y que ciertamente contribuirá en la buena repercusión en los procesos posteriores del tratamiento del mineral.
\end{abstract}

Palabras clave.- Modelamiento, Conminución, Simulación, Optimización, Granulometría, Mineral.

\section{ABSTRACT}

Mathematical modeling applied to equipment's utilized in ore comminution process (crusbing, milling) is so important tool for simulation and optimisation of continuos metallurgical processes (at indudtrial rate), is equivalent, to predict the granulometric yield of a processed ore, by only knowing of feed rate (simulated), obvionsly the model will store intrinsic ly and inherently the plysical properties of ore (hardness, fracture) and equipment operation conditions (CSS opening). The better performance obtained in this manner of the equipment will certaimly contribute for a good consequence in future ore processing worhs.

Key words.- Modeling, Comminution, Simulation, Optimization, Granulometriy, Ore.

\section{INTRODUCCIÓN}

En el presente estudio nos ocuparemos del proceso de conminución en la etapa del chancado (trituración), analizando el desempeño de los diversos equipos como chancadoras de quijadas, giratorias, cónicas: estándar o de cabeza corta short head. Y mediante el uso adecuado del modelamiento; poder determinar el rango operativo que le compete (optimización), al equipo en mención. [1]. Basándonos en ciertos conceptos y funciones matemáticas involucradas en balance poblacional y probabilístico, quienes además constituirán el modelamiento matemático, siendo este a su vez el fiel reflejo del acontecer real manifestado en eventos continuos y constantes en procesos metalúrgicos industriales. Así mismo cabe mencionar a la función clasificación y la función fractura principalmente, que serán tratadas y analizadas en el transcurso del tema a desarrollarse [5].

\footnotetext{
${ }^{1}$ Ing. Estadístico del Área de Ciencias y docente de la Facultad de Ingeniería Geológica, Minera y Metalúrgica de la Universidad Nacional de Ingeniería, ${ }^{2}$ Lic. En Física, Ing. docente de la Facultad de Ingeniería Geológica, Minera y Metalúrgica de la Universidad Nacional de Ingeniería, ${ }^{3}$ Profesor investigador, Ingeniero Metalúrgico de la Universidad Nacional de Ingeniería, ${ }^{4} \mathrm{MSc}$. Ing. docente de la Facultad de Ingeniería Ambiental de la Universidad Nacional de Ingeniería.
} 


\section{DESCRIPCIÓN DEL MODELAMIENTO A SEGUIR}

Se muestra a continuación el significado de las siguientes simbologías a ser utilizadas. [3 y 4].

- $\bar{f}, \bar{p}$ : distribución granulométrica de la alimentación y producto en un proceso de conminución, expresado en una matriz columna: $n x 1$

- $\bar{C}$ : función clasificación, matriz diagonal de tamaño: $n x n$.

- $\bar{B}$ : función fractura acumulada, matriz triangular inferior de tamaño: $n x n$.

- $\bar{b}$ : función fractura parcial, matriz triangular inferior de tamaño: $n x n$.

- $\quad \bar{I}$ : matriz identidad de tamaño: $n x n$.

- $\bar{x}$ : matriz de proceso (que depende de las funciones $\bar{b}$ y $\bar{C}$ ), de tamaño: $n x n$. La denominación $n$ quedará definida o limitada por la variedad o cantidad de tamaño de mallas granulométricas consideradas.

- Amerita también precisar las siguientes matrices y funciones (manifestaciones matemáticas), que intervendrán en el proceso de conminución de minerales:

\section{Matriz columna:}

Alimento: $\bar{f} \quad$ Producto: $\bar{p}$

$$
\bar{f}=\left|\begin{array}{c}
f_{11} \\
f_{21} \\
f_{31} \\
\cdot \\
\cdot \\
\cdot \\
f_{n 1}
\end{array}\right|_{n x 1} \quad ; \quad \bar{p}=\left|\begin{array}{c}
p_{11} \\
p_{21} \\
p_{31} \\
\cdot \\
\cdot \\
\cdot \\
p_{n 1}
\end{array}\right|_{n x 1}
$$

\section{Función clasificación: $C_{i}$}

Referido básicamente a los eventos continuos y constantes que acontece en la etapa del chancado (o de fragmentación), por efecto de la chancadora, hacia los fragmentos $o$ partícula mineral alimentado, con la probabilidad de ser clasificado para su posterior fracturamiento [5]. También nos indica la probabilidad que tiene una partícula de cierto tamaño de ingresar en la etapa de fragmentación dentro de la chancadora.

$$
C_{i}=\left\{\begin{array}{cc}
1-\left(\frac{d_{2}-d_{p i}}{d_{2}-d_{1}}\right)^{n} & d_{1} \prec d_{p i} \prec d_{2} \\
0 & d_{p i} \leq d_{1} \\
1 & d_{p i} \geq d_{2}
\end{array}\right.
$$

donde:

$$
\begin{aligned}
& d p_{i}: \text { tamaño }- \text { promedio }-d e-\text { abertura - } \\
& \text { malla } .(\mathrm{mm}) \\
& d_{1}=\operatorname{CSS} \cdot \alpha_{1} \\
& d_{2}=\text { CSS } . \alpha_{2}+d^{*} \\
& \text { CSS : abertura }-d e-l a-\text { chancadora } .
\end{aligned}
$$

además:

$\left(\Theta_{C}\right)=\left|\alpha_{1}, \alpha_{2}, n, d *\right|$ : Parámetros que cada uno tomará su valor arbitrario, dentro de su rango ya establecido experimentalmente [3,4 y 5].

$$
\begin{aligned}
& 0 \leq \alpha_{1} \leq 1 \\
& 0.1 \leq \alpha_{2} \leq 10 \\
& 0.1 \leq n \leq 10 \\
& d^{*} \geq 0
\end{aligned}
$$

La denominación $n$ en la clasificación es diferente a $n$ relacionado a tamaños granulométricos.

Matricialmente:

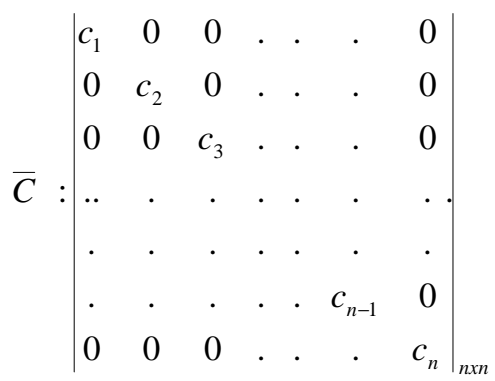




\section{Función fractura acumulada: $\mathbf{B}_{(\mathbf{x}, \mathbf{y})}$}

Esta función nos representa proporcionalmente las partículas de tamaño inicial ' $y$ '; y que luego aparecen en la gama granulométrica de tamaño menor a ' $x$ ', después de la fragmentación. Además la distribución granulométrica obtenida después de la fragmentación de una partícula, es independiente del tamaño inicial de la misma [4].

$$
B_{(x y)}=\left\{\begin{array}{rl}
k\left(\frac{x}{y}\right)^{n_{1}}+(1-k)\left(\frac{x}{y}\right)^{n_{2}} & x \prec y \\
1 & x \geq y
\end{array}\right.
$$

donde:

$x$ : tamaño - granulomet rico - menor.$\left(D_{i}\right)$

$y$ : tamaño - granulomet rico - promedio.$\left(d_{p i}\right)$

además:

$\left(\Theta_{B}\right)=\left|k, n_{1}, n_{2}\right|$ : Parámetros que cada uno tomará su valor arbitrario, dentro de su rango ya establecido experimentalmente. [3,4 y 5].

$$
\begin{aligned}
& 0 \leq k \leq 1 \\
& 0 \leq n_{1} \leq 10 \\
& 0 \leq n_{2} \leq 10
\end{aligned}
$$

Matricialmente:

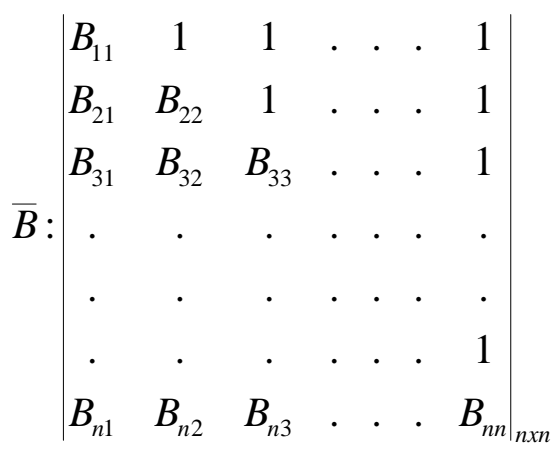

\section{Función fractura parcial: b}

Función fractura o fracción de partículas que aparecen en el intervalo de tamaños i proveniente de la reducción de material del intervalo de tamaño j. [5].
La matriz $\bar{b}$ se calculará por la siguiente ecuación:

$$
\bar{b}=\overline{R^{\prime}}[\overline{\text { ones }}-\bar{B}]
$$

Donde:

$$
\overline{R^{\prime}}=\left|\begin{array}{ccccc}
1 & 0 & \cdot & \cdot & 0 \\
-1 & 1 & \cdot & \cdot & \cdot \\
0 & -1 & . & \cdot & \cdot \\
\cdot & \cdot & \cdot & 1 & 0 \\
0 & 0 & . & -1 & 1
\end{array}\right|_{n x n}
$$

$$
\overline{\text { ones }}=\left|\begin{array}{ccccccc}
1 & 1 & 1 & 1 & . & . & 1 \\
1 & 1 & 1 & . & . & . & 1 \\
1 & 1 & . & . & . & . & . \\
. & . & . & . & . & 1 & 1 \\
1 & 1 & 1 & . & . & 1 & 1
\end{array}\right|_{n x n}
$$

Matricialmente:

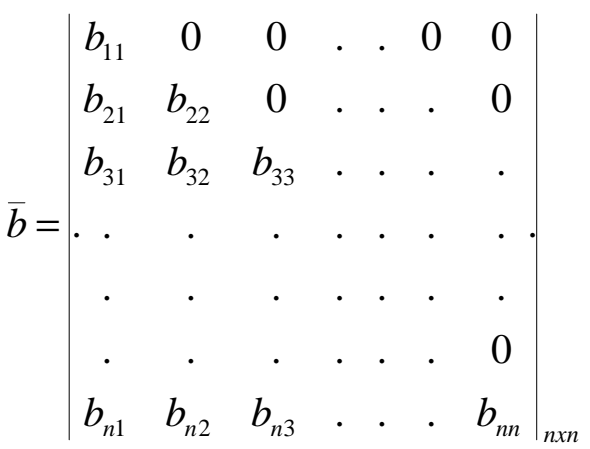

\section{Matriz de proceso: $\bar{x}$}

Donde confluyen las funciones de $\bar{C}$ y $\bar{B}$ principalmente.

Matricialmente: 


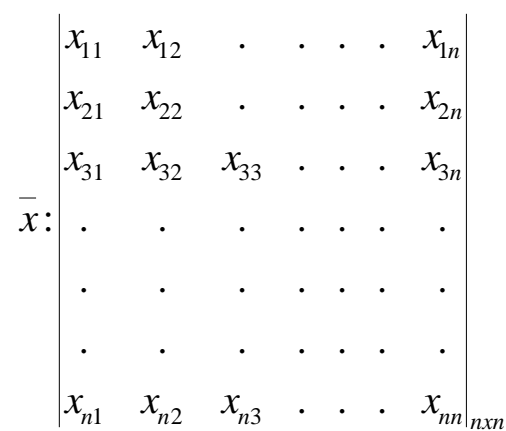

\section{Modelamiento matemático a seguir}

Dentro del equipo (chancadora-trituradora), se produce un sub proceso de clasificación interna y ruptura, como una manifestación del modelamiento a través de un circuito cerrado, donde interviene la alimentación $(\bar{f})$, y el producto $(\bar{p})$, granulométrico, como una combinación de clasificación y fractura de las partículas minerales [6].

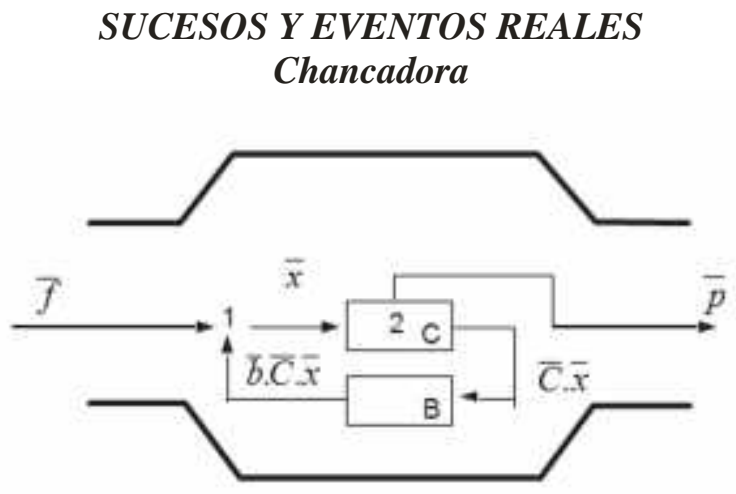

Fig. 1 Circuito de chancado.

Del circuito de chancado (Fig 1), tenemos:

En el nodo 1:

$$
\bar{x}=(\bar{I}-\bar{b} \cdot \bar{C}) \bar{f}
$$

En el nodo 2:

$$
(\bar{I}-\bar{C}) \bar{x}=\bar{p})
$$

De (1) y (2):

$$
\bar{p}=(\bar{I}-\bar{C})(\bar{I}-\bar{b} \cdot \bar{C})^{-} \cdot \bar{f}
$$

La relación (3), expresa el modelamiento matemático, quien involucra a las funciones de clasificación y fractura principalmente. Además serán quienes fortalezcan y generen la consistencia deseada en el proceso de conminución. También dicho modelo estático quedará expresado de la siguiente manera. [2,3 y 4].

$$
\bar{p}=\bar{x} \cdot \bar{f}
$$

Definiendo completamente el proceso de conminución en la etapa de chancado.

\section{APLICACIÓN INDUSTRIAL}

Datos reportados de la etapa de chancado primario con la distribución granulométrica del alimento $(\bar{f})$, del producto $(\bar{p})$ y el $C S S=19.05 \mathrm{~mm}$, según la Tabla 1. [7]. 
Tabla 1. Distribución granulométrica.

\begin{tabular}{|c|c|c|c|c|c|c|}
\hline \multirow{3}{*}{ I.T } & \multirow[b]{3}{*}{ Malla } & \multicolumn{3}{|c|}{ Tamaño (mm) } & \multirow{2}{*}{\multicolumn{2}{|c|}{ Porcentaje en peso (Data) }} \\
\hline & & Maximo & Minimo & Promedio & & \\
\hline & & Di-1 & $\mathrm{Di}$ & dpi & Alimento : $f(x) i$ & Producto : $\mathrm{p}(\mathrm{x}) \mathrm{i}$ \\
\hline 1 & $-4 " / 3 "$ & 101.600 & 76.200 & 87.988 & 15.20 & 0.00 \\
\hline 2 & $-3 "$ / 2" & 76.200 & 50.800 & 62.217 & 27.70 & 0.00 \\
\hline 3 & $-2 " / 1 "$ & 50.800 & 25.400 & 35.921 & 30.30 & 3.80 \\
\hline 4 & $-1 " / 3 / 4 "$ & 25.400 & 19.050 & 21.997 & 11.20 & 13.50 \\
\hline 5 & $-3 / 4 " / 1 / 2 "$ & 19.050 & 12.700 & 15.554 & 4.90 & 20.60 \\
\hline 6 & -1/2" / 3/8" & 12.700 & 9.525 & 10.999 & 3.10 & 20.70 \\
\hline 7 & $-3 / 8 "$ + m3 & 9.525 & 6.680 & 7.977 & 1.90 & 9.60 \\
\hline 8 & $-\mathrm{m} 3+\mathrm{m} 4$ & 6.680 & 4.699 & 5.603 & 1.00 & 5.50 \\
\hline 9 & $-m 4+m 6$ & 4.699 & 3.327 & 3.954 & 0.70 & 4.80 \\
\hline 10 & $-m 6+m 8$ & 3.327 & 2.362 & 2.803 & 0.30 & 2.40 \\
\hline 11 & $-m 8+m 10$ & 2.362 & 1.651 & 1.975 & 0.50 & 3.30 \\
\hline 12 & $-\mathrm{m} 10+\mathrm{m} 14$ & 1.651 & 1.168 & 1.389 & 0.40 & 2.30 \\
\hline 13 & $-\mathrm{m} 14+\mathrm{m} 20$ & 1.168 & 0.833 & 0.986 & 0.30 & 1.90 \\
\hline 14 & $-\mathrm{m} 20+\mathrm{m} 28$ & 0.833 & 0.589 & 0.700 & 0.20 & 1.60 \\
\hline 15 & $-m 28+m 35$ & 0.589 & 0.417 & 0.496 & 0.20 & 1.50 \\
\hline 16 & $-m 35+m 48$ & 0.417 & 0.295 & 0.351 & 0.20 & 1.30 \\
\hline 17 & $-m 48+m 65$ & 0.295 & 0.208 & 0.248 & 0.20 & 0.70 \\
\hline 18 & $-\mathrm{m} 65+\mathrm{m} 100$ & 0.208 & 0.147 & 0.175 & 0.30 & 1.40 \\
\hline 19 & $-m 100+m 150$ & 0.147 & 0.104 & 0.124 & 0.20 & 0.90 \\
\hline 20 & $-m 150+m 20$ & 0.104 & 0.074 & 0.088 & 0.10 & 0.60 \\
\hline 21 & $-\mathrm{m} 200$ & 0.074 & 0.000 & 0.062 & 1.10 & 3.60 \\
\hline
\end{tabular}

De acuerdo a la cantidad de mallas utilizadas en la distribución granulométrica del alimento y producto quedará definido el tamaño matricial de las funciones que intervienen en el modelamiento matemático; es decir para $n$ igual a 21 .

\section{Constituyendo las siguientes: matriz-función}

PRIMERO: De la Tabla 1, obtenemos la función $\bar{f}$.

$$
\bar{f}=\left|\begin{array}{c}
15.20 \\
27.70 \\
30.30 \\
\cdot \\
\cdot \\
\cdot \\
1.10
\end{array}\right|_{21 x 1}
$$

SEGUNDO: Asumiendo en las siguientes funciones de $\bar{C}$ y $\bar{B}$, los siete valores arbitrarios, obviamente dentro de su rango respectivo. [3 y 4].

\section{Función clasificación: $\bar{C}$}

Siendo: $\alpha_{1}=0.725, \alpha_{2}=2.6, n=2, d^{*}=0$

De acuerdo al valor de los cuatro parámetros arbitrarios tomados para la función $\bar{C}$, su expresión matricial será el siguiente: 
$\bar{C}$ :

\begin{tabular}{|lllllllllllllllllllll|}
\hline 1 & 0 & 0 & 0 & 0 & 0 & 0 & 0 & 0 & 0 & 0 & 0 & 0 & 0 & 0 & 0 & 0 & 0 & 0 & 0 & 0 \\
0 & 1 & 0 & 0 & 0 & 0 & 0 & 0 & 0 & 0 & 0 & 0 & 0 & 0 & 0 & 0 & 0 & 0 & 0 & 0 & 0 \\
0 & 0 & 0.854836189 & 0 & 0 & 0 & 0 & 0 & 0 & 0 & 0 & 0 & 0 & 0 & 0 & 0 & 0 & 0 & 0 & 0 & 0 \\
0 & 0 & 0 & 0.405826693 & 0 & 0 & 0 & 0 & 0 & 0 & 0 & 0 & 0 & 0 & 0 & 0 & 0 & 0 & 0 & 0 & 0 \\
0 & 0 & 0 & 0 & 0.0952115091 & 0 & 0 & 0 & 0 & 0 & 0 & 0 & 0 & 0 & 0 & 0 & 0 & 0 & 0 & 0 & 0 \\
0 & 0 & 0 & 0 & 0 & 0 & 0 & 0 & 0 & 0 & 0 & 0 & 0 & 0 & 0 & 0 & 0 & 0 & 0 & 0 & 0 \\
0 & 0 & 0 & 0 & 0 & 0 & 0 & 0 & 0 & 0 & 0 & 0 & 0 & 0 & 0 & 0 & 0 & 0 & 0 & 0 \\
0 & 0 & 0 & 0 & 0 & 0 & 0 & 0 & 0 & 0 & 0 & 0 & 0 & 0 & 0 & 0 & 0 & 0 & 0 & 0 & 0 \\
0 & 0 & 0 & 0 & 0 & 0 & 0 & 0 & 0 & 0 & 0 & 0 & 0 & 0 & 0 & 0 & 0 & 0 & 0 & 0 & 0 \\
0 & 0 & 0 & 0 & 0 & 0 & 0 & 0 & 0 & 0 & 0 & 0 & 0 & 0 & 0 & 0 & 0 & 0 & 0 & 0 & 0 \\
0 & 0 & 0 & 0 & 0 & 0 & 0 & 0 & 0 & 0 & 0 & 0 & 0 & 0 & 0 & 0 & 0 & 0 & 0 & 0 & 0 \\
0 & 0 & 0 & 0 & 0 & 0 & 0 & 0 & 0 & 0 & 0 & 0 & 0 & 0 & 0 & 0 & 0 & 0 & 0 & 0 \\
0 & 0 & 0 & 0 & 0 & 0 & 0 & 0 & 0 & 0 & 0 & 0 & 0 & 0 & 0 & 0 & 0 & 0 & 0 & 0 & 0 \\
0 & 0 & 0 & 0 & 0 & 0 & 0 & 0 & 0 & 0 & 0 & 0 & 0 & 0 & 0 & 0 & 0 & 0 & 0 & 0 & 0 \\
0 & 0 & 0 & 0 & 0 & 0 & 0 & 0 & 0 & 0 & 0 & 0 & 0 & 0 & 0 & 0 & 0 & 0 & 0 & 0 & 0 \\
0 & 0 & 0 & 0 & 0 & 0 & 0 & 0 & 0 & 0 & 0 & 0 & 0 & 0 & 0 & 0 & 0 & 0 & 0 & 0 & 0 \\
0 & 0 & 0 & 0 & 0 & 0 & 0 & 0 & 0 & 0 & 0 & 0 & 0 & 0 & 0 & 0 & 0 & 0 & 0 & 0 & 0 \\
0 & 0 & 0 & 0 & 0 & 0 & 0 & 0 & 0 & 0 & 0 & 0 & 0 & 0 & 0 & 0 & 0 & 0 & 0 & 0 & 0 \\
0 & 0 & 0 & 0 & 0 & 0 & 0 & 0 & 0 & 0 & 0 & 0 & 0 & 0 & 0 & 0 & 0 & 0 & 0 & 0 \\
0 & 0 & 0 & 0 & 0 & 0 & 0 & 0 & 0 & 0 & 0 & 0 & 0 & 0 & 0 & 0 & 0 & 0 & 0 & 0 & 0 \\
0 & 0 & 0 & 0 & 0 & 0 & 0 & 0 & 0 & 0 & 0 & 0 & 0 & 0 & 0 & 0 & 0 & 0 & 0 & 0 & 0 \\
\hline
\end{tabular}

Y gráficamente se manifiesta:

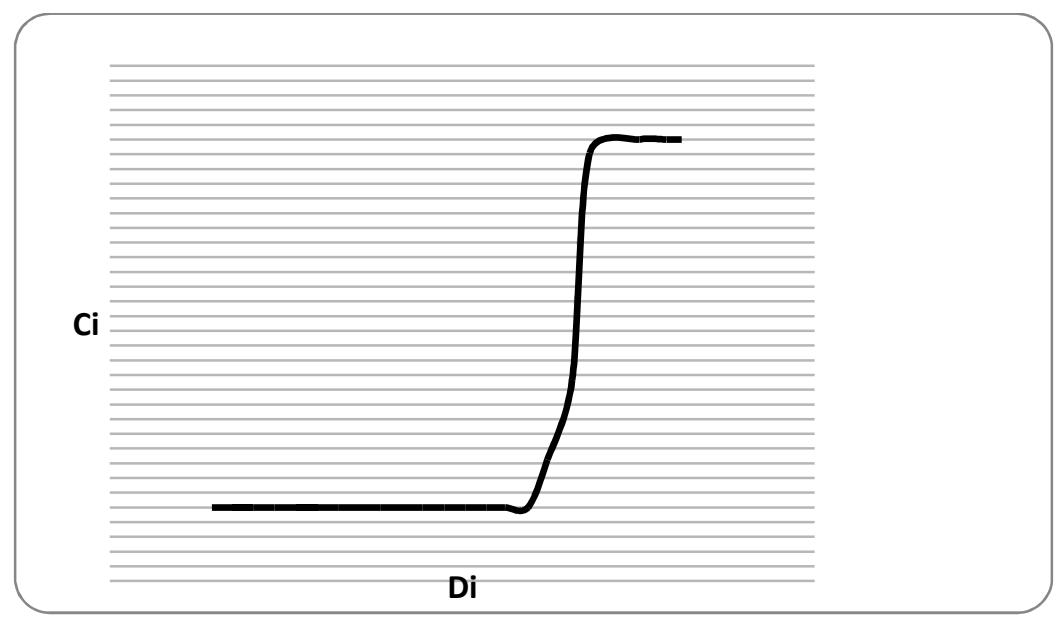

Fig 2. Función clasificación.

\section{Interpretación: Fig. 2}

En la etapa del chancado precisamente en el alimento, sucede que las partículas tan pequeñas respecto a la abertura de descarga de la chancadora no sufrirán ninguna alteración (no fragmentados), por lo que se denota con un valor cero (0); así mismo fragmentos tan grandes ante la abertura, de todas maneras serán fragmentadas equivalente a un valor de uno (1).Y finalmente las partículas de tamaño intermedio, que por su forma aplanada ó alargada y ligada o los eventos de fragmentación, tendrán la probabilidad de ser fragmentados [1].

Función fractura acumulada: $\bar{B}$

Siendo: $k=0.5, n_{1}=0.5, n_{2}=3.5$

Considerando los tres parámetros arbitrarios para la función $\bar{B}$, se consigue constituir la expresión matricial siguiente: 
$\bar{B}$ :

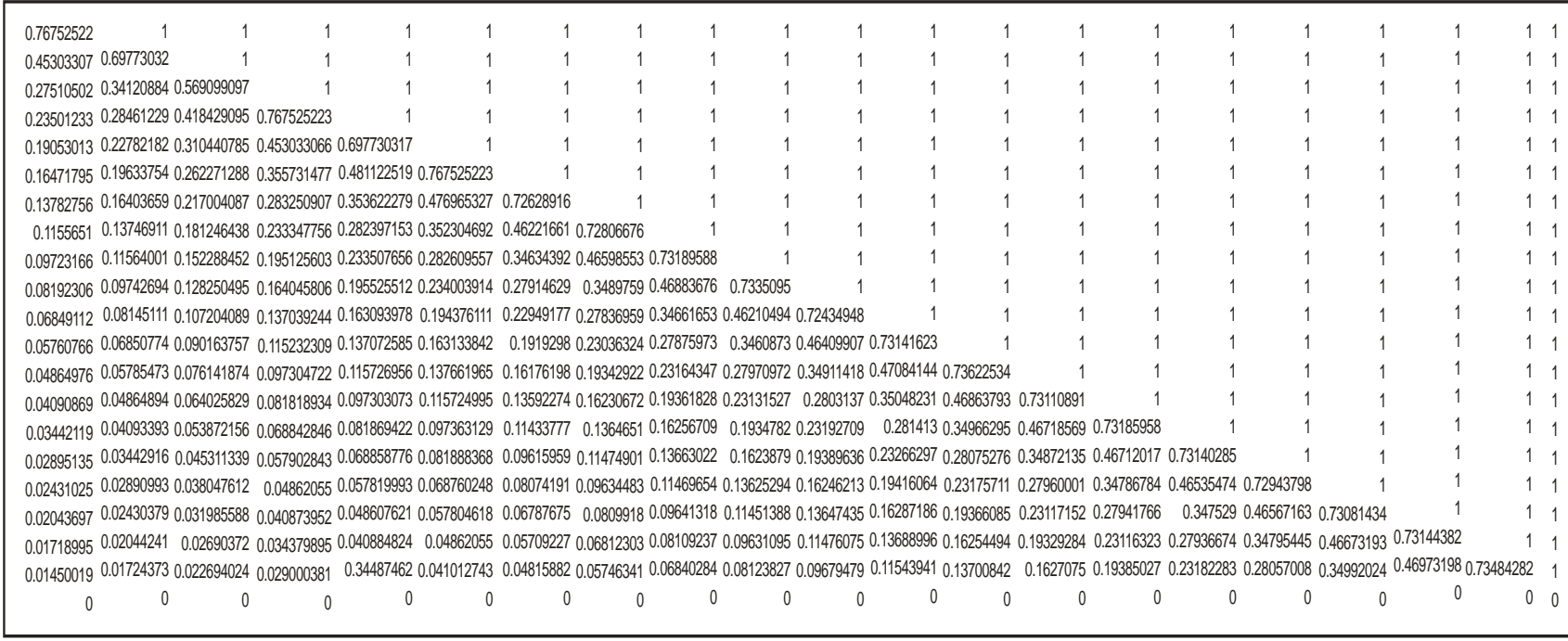

Y graficamente tenemos:

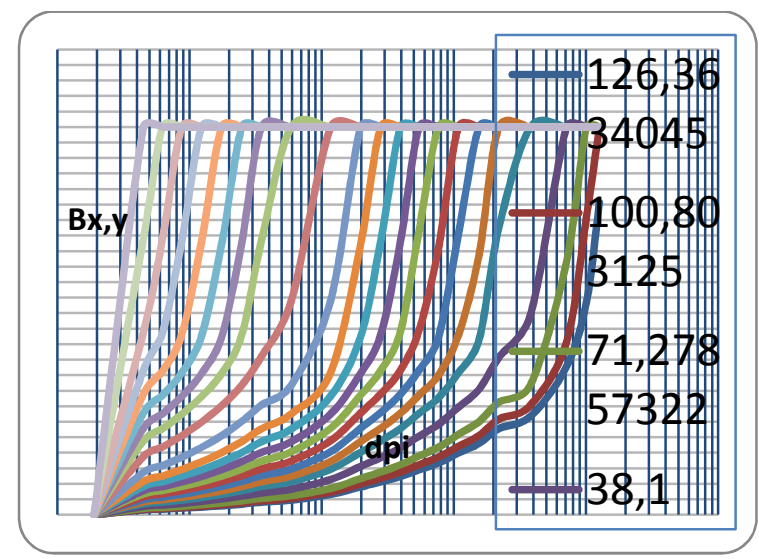

Fig. 3 Función fractura.

\section{Interpretación: Fig. 3}

Los fragmentos grandes a intermedios suelen ser fracturados, generalmente lo hacen en más de un evento $y$ en la continuidad se irán complementando y distribuyendo de acuerdo al grado de tamaños generados en tal proceso de conminución. [3 y 4].

\section{Función fractura fraccionada: $\bar{b}$}

Función que es dependiente de la función $\bar{B}$ principalmente.

Matricialmente tenemos: 
$\bar{b}$ :

\begin{tabular}{|c|c|c|c|c|c|c|c|c|c|c|c|c|c|c|c|c|c|c|c|c|c|c|}
\hline 0.23247478 & 0 & 0 & 0 & 0 & 0 & 0 & 0 & 0 & 0 & 0 & 0 & 0 & 0 & 0 & 0 & 0 & 0 & 0 & 0 & 0 & 0 & 0 \\
\hline 0.31449216 & 0.30226968 & & 0 & 0 & 0 & 0 & 0 & 0 & 0 & 0 & 0 & 0 & 0 & 0 & 0 & b & 0 & 0 & 0 & 0 & 0 & 0 \\
\hline 0.17792805 & 0.35652148 & 80.43090090 & & 0 & 0 & 0 & 0 & 0 & 0 & 0 & 0 & 0 & 0 & 0 & 0 & D & 0 & 0 & 0 & 0 & 0 & 0 \\
\hline 0.04009269 & 0.05659654 & 40.15067000 & 020.232474 & & 0 & 0 & 0 & 0 & 0 & 0 & 0 & 0 & 0 & 0 & 0 & b & 0 & 0 & 0 & 0 & 0 & 0 \\
\hline 0.0444822 & 0.05679047 & 70.1079883 & 310.314492 & 21570.3022696 & & 0 & 0 & 0 & 0 & 0 & 0 & 0 & 0 & 0 & 0 & D & 0 & 0 & 0 & 0 & 0 & 0 \\
\hline 0.02581219 & 0.03148429 & 90.04816949 & 980.0973015 & $589 \mid 0.2166077$ & 77980.2 & 232474777 & 0 & 0 & 0 & 0 & 0 & 0 & 0 & 0 & 0 & b & 0 & 0 & 0 & 0 & 0 & 0 \\
\hline 0.02689039 & 0.03230094 & $4 \quad 0.045267$ & $72 \quad 0.07248$ & $3057 \quad 0.127500$ & 00240.2 & 290559895 & 0.27371084 & 0 & 0 & 0 & 0 & 0 & 0 & 0 & 0 & 0 & 0 & 0 & 0 & 0 & 0 & 0 \\
\hline 0.02226246 & 0.02656749 & 90.03575764 & 490.04990 & 03150.0712251 & 51260.1 & 124660635 & 0.26407255 & 0.27193324 & 0 & 0 & 0 & 0 & 0 & 0 & 0 & b & 0 & 0 & 0 & 0 & 0 & 0 \\
\hline 0.01833344 & 0.02182909 & 90.02895798 & 860.038222 & 21530.0488894 & 94970.0 & 069695136 & 0.11587269 & 0.26208124 & 0.26810412 & 0 & 0 & 0 & 0 & 0 & 0 & 0 & 0 & 0 & 0 & 0 & 0 & 0 \\
\hline 0.0153086 & 0.01821308 & 80.02403795 & 570.031079 & 97970.0379821 & 21440.0 & 048605643 & 0.06719763 & 0.11700963 & 0.26305912 & 0.2664905 & 0 & 0 & 0 & 0 & 0 & 0 & 0 & 0 & 0 & 0 & 0 & 0 \\
\hline 0.01343195 & 0.01597583 & 30.02104640 & 070.027006 & 55630.0324315 & 15340.0 & 039627803 & 0.04965452 & 0.0706063 & 0.12222024 & 0.27140456 & 0.27565052 & 0 & 0 & 0 & 0 & 0 & 0 & 0 & 0 & 0 & 0 & 0 \\
\hline 0.01088345 & 0.01294337 & 0.01704033 & 320.021806 & 69350.0260213 & 13930.0 & 031242268 & 0.03756197 & 0.04800635 & 0.06785679 & 0.11601621 & 0.26025041 & 0.26858377 & 0 & 0 & 0 & 0 & 0 & 0 & 0 & 0 & 0 & 0 \\
\hline 0.0089579 & 0.01065301 & 0.01402188 & 820.017927 & 75870.0213456 & 56290.0 & 025471878 & 0.03016782 & 0.03693402 & 0.04711626 & 0.6637901 & 0.11498489 & 0.26057478 & 0.26377466 & 0 & 0 & D & 0 & 0 & 0 & 0 & 0 & 0 \\
\hline 0.00774107 & 0.00920579 & 0.01211604 & 460.015482 & 27880.0184238 & 38830. & 0.02193697 & 0.02583925 & 0.03112251 & 0.03802519 & 0.04839445 & 0.06880048 & 0.12035914 & 0.2675874 & 0.26889109 & 0 & 0 & 0 & 0 & 0 & 0 & 0 & 0 \\
\hline 0.0064875 & 0.007715 & 0.01015367 & 730.012976 & 60880.0154336 & 36510.0 & 018361866 & 0.02158497 & 0.02584162 & 0.03105119 & 0.03783707 & 0.0483866 & 0.06906931 & 0.11897498 & 0.26392322 & 0.26814042 & & 0 & 0 & 0 & 0 & 0 & 0 \\
\hline 0.00546984 & 0.00650478 & 30.00856081 & 170.010940 & 00030.0130106 & 06460.0 & 015474761 & 0.01817818 & 0.02171609 & 0.02593686 & 0.0310903 & 0.03803073 & 0.04875003 & 0.06891019 & 0.11846434 & 0.26473941 & 0.2685971 & 15 & 0 & 0 & 0 & 0 & 0 \\
\hline 0.0046411 & 0.00551923 & 30.00726372 & 270.009282 & 22930.0110387 & 87830. & 0.01312812 & 0.01541768 & 0.01840417 & 0.02193368 & 0.02613496 & 0.03143424 & 0.03850233 & 0.04899565 & 0.06912134 & 0.11925233 & & & & 0 & 0 & 0 & 0 \\
\hline 0.00387328 & 0.00460614 & 0.00606202 & 230.007746 & 05980.0092123 & 23720. & 0.01095563 & 0.01286516 & 0.01535303 & 0.01828336 & 0.02173906 & 0.02598778 & 0.03128878 & 0.03809626 & 0.04842849 & 0.06845017 & 70.1178257 & & 6350.2691856 & & 0 & 0 & 0 \\
\hline 0.00324702 & 0.00386138 & 0.00508186 & 690.006494 & 40570.0077227 & 27970.0 & 009184068 & 0.01078448 & 0.01286876 & 0.01532081 & 0.01820292 & 0.0217136 & 0.0259819 & 0.03111591 & 0.03787868 & 0.04825443 & 30.0681622 & 260.1177171 & 7180.2640824 & 2410.268556 & 5618 & 0 & 0 \\
\hline 0.00268976 & 0.00319868 & 30.00420969 & 960.005379 & 95140.0063973 & 73620.0 & 007607806 & 0.00893344 & 0.01065962 & 0.01268953 & 0.01507268 & 0.01796596 & 0.02145055 & 0.02553653 & 0.03058534 & 0.03731296 & 0.0475439 & 9910.0673843 & 1370.1168116 & 1690.261711 & 11830.2651 & 5718 & 0 \\
\hline 0.01450019 & 0.01724373 & 30.02269402 & 240.029000 & 03810.0344874 & 74620.0 & 041012743 & 0.04815882 & 0.05746341 & 0.06840284 & 0.08123827 & 0.09679479 & 0.11543941 & 0.13700842 & 0.1627075 & 0.19385027 & 0.2318228 & 830.2805700 & 0080.3499202 & 0240.469731 & 31980.7348 & 4282 & 1 \\
\hline
\end{tabular}

Matriz de Proceso: $\bar{X}$

Matricialmente:

Función que es dependiente de las funciones: $\bar{C}$ y

$\bar{B}$ respectivamente [1, 3 y 4 ].

$\bar{X}$ :

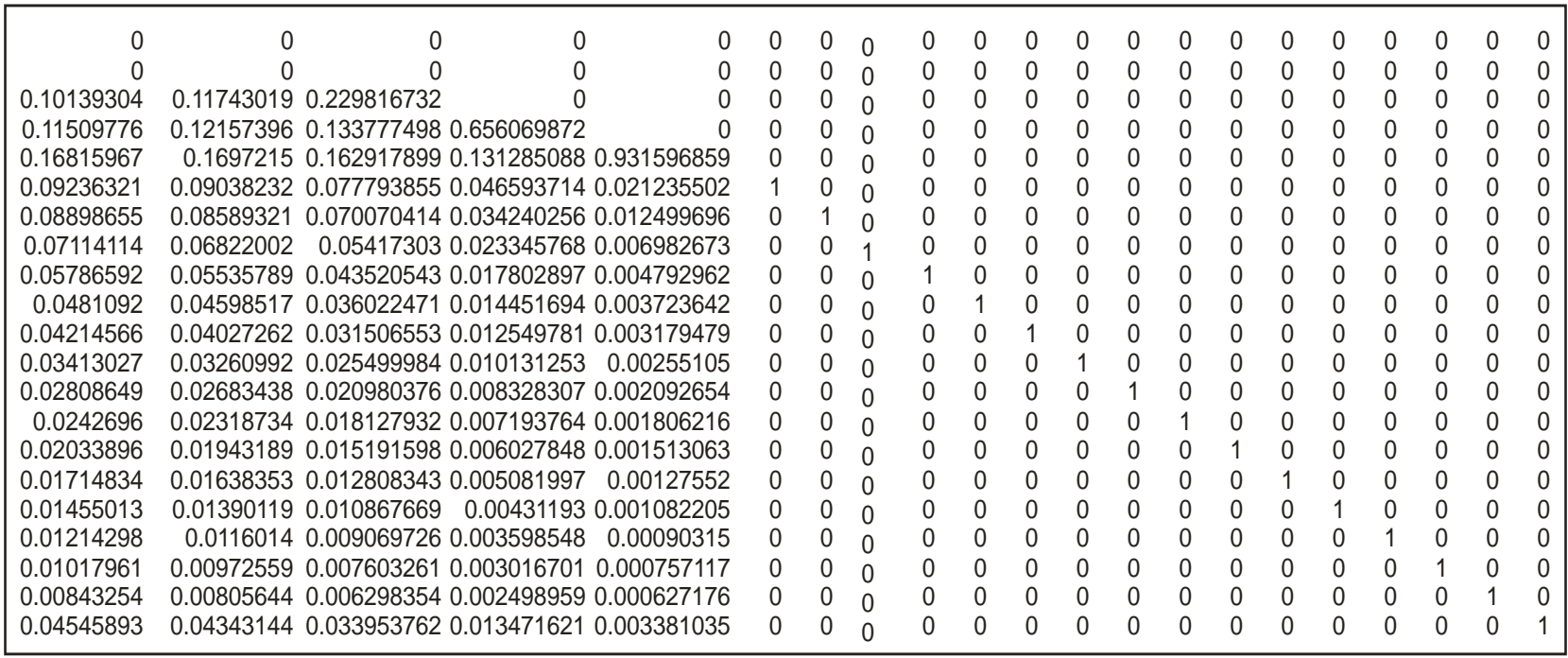

Si sustituimos en la relación (4) los valores de las funciones de $\bar{x}$ y $\bar{f}$, el modelo matemático me arroja un producto granulométrico (ver Tabla 2), observándose cierta divergencia comparativa respecto a la data real. Así mismo se visualiza la tendencia hacia lo real (ver Fig. 4), con error equivalente a 0.01972985 . 
Mercedes Aida Osorio Maza, Zoila Emperatriz Ruiz Mostacero, Edgar Ademar Pérez Matos, Luis Alvarado Jaramillo.

Tabla 2. Datos comparativos.

\begin{tabular}{|c|c|c|}
\hline $\begin{array}{c}\text { Tamaño } \\
\text { promedio: } d p i\end{array}$ & $\begin{array}{c}\text { Producto: } \boldsymbol{D} \boldsymbol{F} \\
\boldsymbol{F}(\boldsymbol{x})\end{array}$ & $\begin{array}{c}\text { Producto: } \boldsymbol{M d} \\
\boldsymbol{F}(\boldsymbol{x})\end{array}$ \\
\hline 87.988 & 1.000 & 1.000000000 \\
\hline 62.217 & 1.000 & 1.000000000 \\
\hline 35.921 & 0.962 & 0.882425026 \\
\hline 21.997 & 0.827 & 0.717240373 \\
\hline 15.554 & 0.621 & 0.534950947 \\
\hline 10.999 & 0.414 & 0.435045262 \\
\hline 7.977 & 0.318 & 0.353048160 \\
\hline 5.603 & 0.263 & 0.293966457 \\
\hline 3.954 & 0.215 & 0.247421197 \\
\hline 2.803 & 0.191 & 0.211654849 \\
\hline 1.975 & 0.158 & 1.177985337 \\
\hline 1.389 & 0.135 & 0.150778392 \\
\hline 0.986 & 0.116 & 0.128683758 \\
\hline 0.700 & 0.100 & 0.110184916 \\
\hline 0.496 & 0.085 & 0.094358449 \\
\hline 0.351 & 0.072 & 0.080701105 \\
\hline 0.248 & 0.065 & 0.068809931 \\
\hline 0.175 & 0.051 & 0.057555193 \\
\hline 0.124 & 0.042 & 0.048635145 \\
\hline 0.088 & 0.036 & 0.041902748 \\
\hline 0.062 & 0.000 & 0.000000000 \\
\hline
\end{tabular}

- Producto Dt: data real reportado.

- Producto Md: data del modelo.

Por el momento solo se ha conseguido vislumbrar cierta tendencia comparativa (de productos granulométricos), al considerar los siete valores arbitrarios.
A continuación se tratará de minimizar el error ya contraído y llegar a establecer una aproximación que se asemeje y represente a lo real, todo aquello mediante el uso de solver-excel.

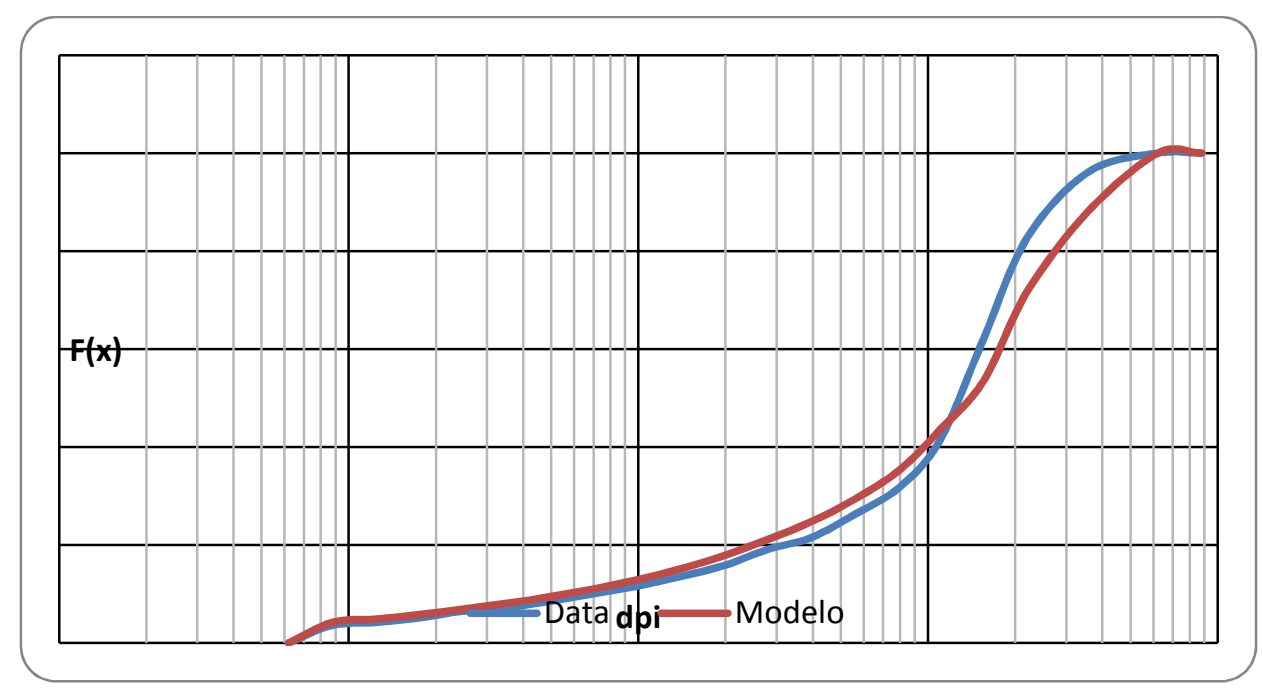

Fig. 4 Tendencia de modelo. 
TERCERO: Utilizando solver-excel conseguimos:

- Que el error tienda a cero y de recalcular los siete parámetros arbitrarios, consecuentemente con sus valores óptimos.
- Que el modelo adquiera una buena aproximación hacia lo real; con un error mínimo de 0.00279

Finalmente poder corroborar la afinidad de los datos (ver Tabla 3) y visualizando la proximidad (ver Fig. 5).

Tabla 3. Corroborando datos.

\begin{tabular}{|c|c|c|}
\hline $\begin{array}{c}\text { Tamaño } \\
\text { promedio:dpi }\end{array}$ & $\begin{array}{c}\text { Producto: } \boldsymbol{D t} \\
\boldsymbol{F}(\boldsymbol{x})\end{array}$ & $\begin{array}{c}\text { Producto: } \boldsymbol{M d} \\
\boldsymbol{F}(\boldsymbol{x})\end{array}$ \\
\hline 87.988 & 1.000 & 1.000000000 \\
\hline 62.217 & 1.000 & 0.999899761 \\
\hline 35.921 & 0.962 & 0.958453149 \\
\hline 21.997 & 0.827 & 0.842008682 \\
\hline 15.554 & 0.621 & 0.610772415 \\
\hline 10.999 & 0.414 & 0.437738468 \\
\hline 7.977 & 0.318 & 0.322158813 \\
\hline 5.603 & 0.263 & 0.256051634 \\
\hline 3.954 & 0.215 & 0.211960346 \\
\hline 2.803 & 0.191 & 0.181430983 \\
\hline 1.975 & 0.158 & 0.153584039 \\
\hline 1.389 & 0.135 & 0.131439782 \\
\hline 0.986 & 0.116 & 0.113528104 \\
\hline 0.700 & 0.100 & 0.098547994 \\
\hline 0.496 & 0.085 & 0.085542034 \\
\hline 0.351 & 0.072 & 0.07413378 \\
\hline 0.248 & 0.065 & 0.064031359 \\
\hline 0.175 & 0.051 & 0.054164524 \\
\hline 0.124 & 0.042 & 0.04631780 \\
\hline 0.088 & 0.036 & 0.040398842 \\
\hline 0.062 & 0.000 & 0.000000000 \\
\hline
\end{tabular}

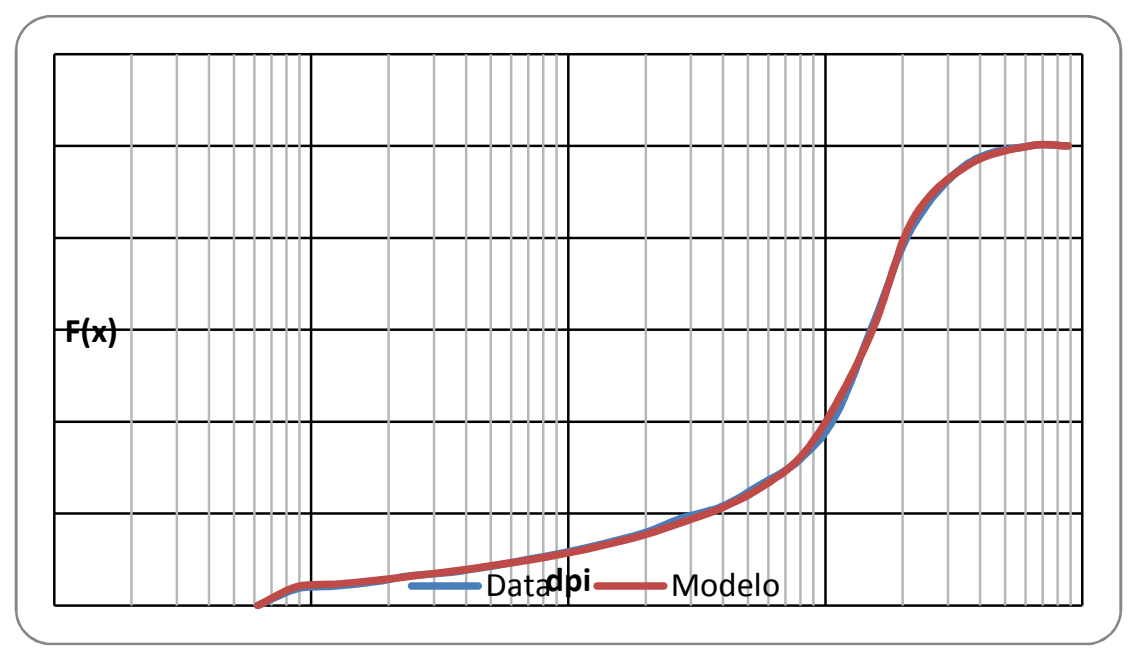

Fig. 5 Aproximación del modelo. 


\section{RESULTADOS Y DISCUSIONES}

Considerando inicialmente los siete valores arbitrarios se consigue vislumbrar cierta tendencia, hacia la data granulométrica real del producto (Fig 4), con un error de 0.01972985 .

Sin embargo, haciendo que los errores por cada tamaño granulométrico sea mínimo se consigue la aproximación deseada con 0.00279 (de error modelo); con lo cual también se obtuvo el recalculo de todos los valores paramétricos óptimos siguientes (mediante solver-excel):

$$
\begin{array}{ll}
k=0.216036 & \alpha_{1}=0.5773503 \\
n_{1}=0.4545568 & \alpha_{2}=5.2417205 \\
n_{2}=2.5960338 & n=10.0000 \\
& d^{*}=0
\end{array}
$$

Además se puede visualizar (Fig 5), la consistencia que adquiere el modelo; pues la proximidad es tal que la curvas correspondientes a la data y el modelo se confunden.

La función de proceso $\bar{x}$ de la relación (4), ampara e involucra a $\bar{C}$ y $\bar{B}$; como una combinación de probabilidad de fracturamiento poblacional a través de un circuito cerrado en la etapa del chancado.

\section{CONCLUCIONES}

Los parámetros hallados en condición mínima de error, adquieren la denominación de valores óptimos, quienes hacen posible que el modelamiento matemático este apto para poder simular (asemejar), y lograr predecir la ocurrencia granulométrica a través del proceso del chancado.

Es decir simular la granulometría de salida de un equipo de conminución, en función de la granulometría de entrada y del proceso de reducción de tamaño que sufre el material dentro del equipo.

Entonces para cada alimento simularemos un producto y en lo sucesivo poder llegar a establecer un rango de estabilidad del desempeño del equipo (optimización en la etapa del chancado).
Así mismo podemos utilizar tal modelo como una herramienta tan importante para monitorear los avances continuos y con la data obtenida poder procesarla, llegando a definir y determinar el desempeño operativo del equipo (chancadoratrituradora), en la etapa de chancado.

\section{AGRADECIMIENTOS}

A los docentes de la facultad de Ingeniería Geológica Minera y Metalúrgica que hicieron posible la culminación del presente trabajo y en especial al MSc. Ing. Oscar Silva Campos y MSc Ing. José Antonio Kobashikawa Chinen, por su permanente apoyo.

\section{REFERENCIAS}

1. Lynch, J. A., Fuerstenau, W. D. "Circuito de trituración y molienda. Desarrollo en los procesos minerales - simulación optimización - diseño y control". Edit. Rocas y Minerales. $1^{\circ}$ Edición 1980.

2. Ayres Hidalgo, F. A., Torres Ponce, M. T. "Técnicas matemáticas aplicadas al balance de materia". Ingeniería y computación EIRL. Arequipa 1998.

3. Leonel Gutiérrez R., Sepúlveda, J. "Dimensionamiento y optimización de plantas concentradoras mediante técnicas de modelación matemática". Centro de investigación minera metalúrgica, 1986.

4. G. Austin, L., Concha A. F., "Diseño y simulación de circuitos de molienda y clasificación". Programa iberoamericano de ciencia y tecnología para el desarrollo. Subprograma de tecnología mineral. Red de fragmentación XII-A.

5. Oblad, A. E., GS technologies / control international, Salt. Lake City, Utah, USA. "Modelos matemáticos de conminución y sus aplicaciones en la industria mineral". Nov.1994.

6. Will, B. A. - Naiper,T. J. "Mineral processing technology". An introduction to the practical aspects of ore treatment and mineral recovery: October 2006.

7. Taggart, A. F. "Elementos de Preparación de minerales". Profesor de Ingeniería de Minerales de la Escuela de Minas de la Universidad de Columbia (Nueva York) 1966. 
Modelamiento matemático aplicado a conminución

Correspondencia:ademaredgar@yahoo.com

Recepción de originales: enero 2014

Aceptación de originales: marzo 2014 